\title{
Synthesis of wind tunnel and climatological data for estimating design wind effects: A copula based approach
}

\author{
Workamaw P. Warsido ${ }^{\text {, Girma T. Bitsuamlak }}{ }^{\mathrm{b}, *}$ \\ ${ }^{a}$ Department of Civil and Environmental Engineering, International Hurricane Research Cneter, \\ Florida International University, 10555 W Flagler St, Miami, Florida 33174, USA \\ ${ }^{b}$ Department of Civil and Environmental Engineering, WindEEE Research Institute, University \\ of Western Ontario in London, ON, Canada N6A 5B9
}

\begin{abstract}
Accurate estimation of design wind effects on tall buildings is critical to ensure adequate margin of safety against lateral wind loads with a reasonable cost. Design wind effects on tall buildings are estimated by combining aerodynamic data obtained from wind tunnel tests with the climatological or wind speed data of the construction site. Though it is widely agreed that the aerodynamic data obtained from wind tunnel tests is fairly reliable, the various approaches used to synthesize aerodynamic and climatological data are often argued to have shortcomings. For instance, the uncertainty in modeling the correlation effect between sectorial responses has always been a challenge in the sector-by-sector synthesis approach. This paper presents a new copula based approach for modeling this correlation effect between sectorial responses. In addition, the proposed approach is used to assess the uncertainties in assuming sectorial responses to be perfectly dependent or independent while using the sector-by-sector approach. Results of the study showed that a copula based approach can be used for accurate modeling of the correlation effect between sectorial responses. It was also noted that assuming sectorial responses to be perfectly dependent or independent could result in underestimation or overestimation of design wind effects respectively.
\end{abstract}

${ }^{*}$ Corresponding author Tel.: +1 (519) 6612111 X 88028, Fax.+1 (519) 661 3339, E-mail: gbitsuam@uwo.ca, 


\section{Keywords: Directionality; Tall building; Copula; Aerodynamic data; Climatological data.}

\section{Introduction}

Wind-induced responses of tall buildings are often direction dependent because of the asymmetrical nature of their geometrical and/or structural properties. Hence, wind tunnel tests on tall buildings are often conducted for multiple wind directions by rotating the building model with respect to a unidirectional wind flow in the tunnel. The aerodynamic responses measured in the wind tunnel are normalized by the product of a reference dynamic pressure and a certain weighting factor computed based on the type of response. Full-scale design wind effects of the required mean recurrence interval (MRI) can then be estimated by integrating the normalized aerodynamic data with the actual wind speed data obtained from the construction site. Besides the aerodynamic data collected from wind tunnel tests, magnitude of the actual wind speed in a given area also shows variation with direction depending on the exposure and climatology of the area. Hence, synthesis of the aerodynamic and climatological data involves considering this wind directionality effect. The wind directionality effect plays a significant role particularly in the design of wind sensitive structures such as tall buildings. It is agreed among many researchers that neglecting wind directionality effect for wind sensitive structures could result in overly conservative design [e.g. 1, 2, 3]. It was also noted in the works of various researchers [e.g. 4, 5] that significant under estimation of design wind effects could result when the wind directionality effect is not properly considered. Thus, for a safe and economical design it is highly recommended that the wind directionality effect is properly considered while synthesizing aerodynamic and climatological data. Several techniques have been suggested over the years for integrating climatological data into the wind tunnel test results and some of these techniques are briefly reviewed as follows. The first and most simplified approach is the directionality factor approach which has been implemented in several design codes and standards. In this approach a 
single wind speed vector is constructed which consists of the largest wind speed in a given epoch irrespective of wind direction. Similarly, the largest peak aerodynamic coefficient out of all wind directions tested in the wind tunnel is extracted. The wind speed vector is combined with the largest aerodynamic coefficient to estimate the required design wind effect. However, the probability of the largest magnitude wind speed coming in the most aerodynamically unfavorable direction is most likely to be less than 1 . In order to account for this reduced probability, some design codes and standards recommend using a directionality factor of less than 1. For instance, ASCE 7-10 recommends a directionality factor of 0.85 to be used for most structures including tall buildings. Some researchers showed that the use of such generic reduction factors may not always give a safe result [e.g. 6, 7]. Upcrossing method is another synthesis technique used to integrate aerodynamic and climatological data. This method was originally suggested by Davenport [8] and later modified by Lepage and Irwin [9]. The basic concept behind the method lies in estimating the mean upcrossing rate of a response boundary by a wind speed, the inverse of which gives the return period corresponding to the given wind effect. The response boundary is a curve which shows variation of the wind speed required to cause a given level of wind effect as a function of wind direction. Further discussion on the formulation of this approach can be referred from [10]. This method is analytically more involved compared to the other approaches reviewed here. In addition, some researchers [e.g. 5, 11] cited the challenges in accurately evaluating some of the parameters defined in the formulation of this approach. Storm passage approach is another technique used to integrate aerodynamic and wind climate data. In this approach, the aerodynamic data obtained from wind tunnel testing is converted into time history of wind effects using hourly recorded directional wind speed data. The time history data is then divided into sub-intervals and extreme value analysis is carried out on the maximum values of 
the sub-intervals to estimate the required level of design wind effect. Further discussion on the formulation of this approach can be referred from $[10,12]$. This method is simple to implement provided sufficiently long and reliable wind speed records are available. In addition, it involves fewer approximations in its formulation compared to the upcrossing method. However, this method is computationally intensive as it involves evaluating hour by hour time history of wind effects. Sufficiently long and reliable wind speed data for every project location may not be readily available as well. Sector-by-sector approach is another method used to synthesize aerodynamic and climatological data. In this approach, the wind speed and aerodynamic data are grouped and synthesized in sectors to get extreme wind effects corresponding to each sector. The final design wind effect will be estimated considering the statistical correlation between the sectorial wind effects. However, proper modeling of this correlation effect between sectorial wind effects has always been a challenge in applying this method. Compared to the upcrossing and storm passage approaches, this method is simple and computationally less intensive. Considering this advantage, this method can be more appealing if the statistical correlation effect between sectorial wind effects can be evaluated more accurately. This paper introduces a new copula based approach for modeling this correlation effect between sectorial wind effects. Formulation of the method will be discussed in detail in the following section followed by a numerical example illustrating its application. In addition, the method will be used to assess the uncertainties in the traditional simplifying assumptions of perfectly dependent and independent sectorial wind effects while implementing the sector-by-sector approach.

\section{Sector-by-sector directional analysis}

In the sector-by-sector synthesis approach the aerodynamic and directional wind speed data are grouped in sectors. Grouping of the data in sectors is required since the wind speed data is often available for limited number of directions compared to the aerodynamic data measured 
in the wind tunnel. For instance, the aerodynamic data is often available for 36 directions whereas the actual wind speed data might be available only for 8 or 16 directions. Thus, the decision on the number of sectors to be used is mostly dictated by the available directional wind speed data. The largest magnitude peak aerodynamic coefficient in each sector is combined with the corresponding sectorial wind speed to get the largest sectorial wind-induced response as follows:

$$
\hat{R}_{i}=\frac{1}{2} \rho_{a} W_{R} \times \hat{C}_{R i} U_{i}^{2}
$$

where $\hat{R}_{i}$ is a vector of peak wind-induced response corresponding to sector $i ; \rho_{a}$ is the density of air; $W_{R}$ is a weighting factor computed based on the type of response; $\hat{C}_{R i}$ is the largest peak aerodynamic response coefficient in sector $i$ and $U_{i}$ is a vector of extreme wind speed corresponding to sector $i$. Extreme value analysis is then carried out to estimate response of the required MRI, say $T$ years, corresponding to sector $i$. If there are $k$ sectors, extreme value analysis is carried out for each sector separately and a total of $k$ sectorial extreme wind-induced responses will be obtained. The question one might ask at this stage would be whether it is possible to take the largest sectorial response $\left(R_{\max }\right)$ as the design response of $T$ years MRI or not. In probability terms, this is the same as asking if the probability of non-exceedance of the response $R_{\max }$ in $T$ years is given by $\operatorname{Pr}\left(R \leq R_{\max }\right)=1-1 / T$. The non-exceedance probability $\operatorname{Pr}\left(R \leq R_{\max }\right)$ can be computed from the joint distribution of sectorial responses which is given as follows:

$$
\operatorname{Pr}\left(R \leq R_{\max }\right)=\operatorname{Pr}\left[\left(R \leq R_{\max }\right)_{1},\left(R \leq R_{\max }\right)_{2}, \ldots\left(R \leq R_{\max }\right)_{k}\right]
$$


where the subscripts $1,2, \ldots, k$ denote sector numbers. If all the sectorial responses are fully correlated (perfectly dependent) the multivariate distribution given in Eq. (2) can be evaluated as:

$$
\operatorname{Pr}\left(R \leq R_{\text {max }}\right)_{d e p}=\min \left[\operatorname{Pr}\left(R \leq R_{\text {max }}\right)_{1}, \operatorname{Pr}\left(R \leq R_{\max }\right)_{2}, \ldots, \operatorname{Pr}\left(R \leq R_{\max }\right)_{k}\right]
$$

where the subscript dep shows the condition of full dependence between sectorial responses and the operation $\min []$ denotes the minimum value. Since the minimum probability of nonexceedence which is $1-1 / T$ is obtained in the sector where the largest sectorial response $\left(R_{\max }\right)$ occurs, Eq. (3) can be written as:

$$
\operatorname{Pr}\left(R \leq R_{\max }\right)_{d e p}=1-1 / T
$$

In contrary, if all the sectorial responses are fully uncorrelated (perfectly independent) the multivariate distribution given in Eq. (2) can be evaluated as the product of sectorial probabilities as follows:

$$
\operatorname{Pr}\left(R \leq R_{\text {max }}\right)_{\text {indep }}=\operatorname{Pr}\left(R \leq R_{\max }\right)_{1} \times \operatorname{Pr}\left(R \leq R_{\max }\right)_{2} \times \ldots \times \operatorname{Pr}\left(R \leq R_{\max }\right)_{k}
$$

where the subscript indep shows the condition of full independence between sectorial responses. Hence, under this condition the probability of non-exceedance $\operatorname{Pr}\left(R \leq R_{\max }\right)$ will be less than the minimum probability $1-1 / T$ since the individual probabilities have values between 0 and 1 .

$$
\operatorname{Pr}\left(R \leq R_{\text {max }}\right)_{\text {indep }}<1-1 / T
$$

However, assuming sectorial responses to be fully dependent or independent may not be realistic since the actual correlation falls between the two extremes. Hence, for accurate estimation of design responses the correlation effect between sectorial responses should be considered properly. As to the authors' knowledge, there hasn't been an explicit multivariate extreme value 
distribution which can be used to combine marginal distributions of multiple sectorial responses considering their statistical correlation. This paper introduces an alternative approach for estimating the joint probability distribution of multiple sectorial responses. This approach uses the concept of "copulas" for modeling the dependence structure between sectorial marginal distributions. Copulas are functions which are used to model the dependence or correlation structure between two or more variables whose marginal distributions are known. The basic principle behind the copula approach is to treat the dependence structure separately from the marginal distributions. Since the dependence information is contained in the copula the marginal distributions need not be from the same family unlike the traditional multivariate analysis approaches. In recent years, copulas have been increasingly used in statistics, finance, economics, risk management, transportation, hydrology and several other fields. A brief discussion for laying out the mathematical background on copulas is given in the following section. Further discussion on copula modeling can be referred from several published literatures [e.g. 13, 14-18].

\section{Modeling dependence with copulas}

\subsection{Definition}

Consider a set of random variables $R_{1}, R_{2}, \ldots, R_{k}$ whose joint and marginal cumulative distribution functions(cdfs) are given by Eqs. (7) and (8) respectively.

$$
\begin{gathered}
F\left(r_{1}, r_{2}, \ldots, r_{k}\right)=\operatorname{Pr}\left(R_{1} \leq r_{1}, R_{2} \leq r_{2}, \ldots, R_{k} \leq r_{k}\right) \\
F_{i}(r)=\operatorname{Pr}\left(R_{i} \leq r\right) \quad i=1,2, \ldots, k
\end{gathered}
$$

From Sklar's theorem[19], for all $r_{i}$ in $[-\infty, \infty], i=1,2, \ldots, k$ there exists a $k$-dimensional copula function $C:[0,1]^{k} \rightarrow[0,1]$ such that 


$$
F\left(r_{1}, r_{2}, \ldots, r_{k}\right)=C\left[F_{1}\left(r_{1}\right), F_{2}\left(r_{2}\right), \ldots, F_{k}\left(r_{k}\right)\right]
$$

Sklar's theorem also states that the $k$ dimensional copula $C$ should at least satisfy the following conditions:

(i) $C\left(1, \ldots, 1, u_{j}, 1, \ldots, 1\right)=u_{j} \quad$ for every $j \leq k$ and all $u_{j}$ in $[0,1]$

(ii) $C\left(u_{1}, u_{2}, \ldots, u_{k}\right)=0 \quad$ if $u_{j}=0$ for any $j \leq k$

(iii) $C$ is $k$-increasing According to the first condition, if the marginal cdf of $k-1$ variables is known to be 1 then the joint cdf of $k$ variables which includes $u_{j}$ is the same as the marginal cdf of $u_{j}$. Based on the second condition, if the marginal cdf of at least one of the $k$ variable is zero then the joint cdf of all the $k$ variables is zero. The third condition shows that the function should be $k$-increasing in order to be a $k$-dimensional copula. A given $k$-place real function $H$ is said to be $k$-increasing if the $H$-volume of $B\left(V_{H}(B)\right)$ is non-negative for every $k$-box $B$ whose vertices lie in the domain of $H[17]$. A $k$-box $B[x, y]$ can be defined by the Cartesian product given in Eq. (10) and its $H$ volume can be computed by the sum given in Eq. (11)

$$
\begin{gathered}
{[x, y]=\left[x_{1}, y_{1}\right] \times\left[x_{2}, y_{2}\right] \times \ldots \times\left[x_{k}, y_{k}\right]} \\
\sum_{w \in v e r[(x, y])} \operatorname{sgn}(w) H(w) \geq 0
\end{gathered}
$$

Any copula $C$ should satisfy the Fréchet-Hoeffding bounds inequality given in the following equation [20].

$$
W\left(u_{1}, u_{2}, \ldots, u_{k}\right) \leq C\left(u_{1}, u_{2}, \ldots, u_{k}\right) \leq M\left(u_{1}, u_{2}, \ldots, u_{k}\right)
$$

where the lower and upper bounds are given by 


$$
W\left(u_{1}, u_{2}, \ldots, u_{k}\right)=\max \left[\sum_{i=1}^{k} u_{i}-k+1,0\right] \text { and } M\left(u_{1}, u_{2}, \ldots, u_{k}\right)=\min \left[u_{1}, u_{2}, \ldots, u_{k}\right]
$$

For bivariate case $(k=2)$, both the lower and upper bounds are copulas while for $k>2$ the lower bound qualifies to be copula only under certain conditions. Detail discussion on these conditions which should be satisfied by any copula can be obtained from several published literatures[e.g. 13, 21]. Based on the criteria discussed so far the joint probabilities of fully dependent and independent sectorial responses given in Eqs. (3) and (5) constitute copulas which are commonly referred as dependent and independent copulas. Different copula functions have been derived over the years for various applications. Archimedean copulas are one of the most widely used copulas for different applications. Gumbel copula which is an Archimedean as well as an extreme value copula is used in the present study and the discussion hereafter will be mostly based on Gumble copula.

\subsection{Archimedian copulas}

Archimedean copulas are those copulas which can be described by the following formulation:

$$
C\left(u_{1}, u_{2}, \ldots, u_{k}\right)=\varphi\left\lfloor\varphi^{-1}\left(u_{1}\right)+\varphi^{-1}\left(u_{2}\right)+\ldots+\varphi^{-1}\left(u_{k}\right)\right\rfloor
$$

where $\varphi$ is the copula generator function. For instance, the copula generator function for Gumble copula is given by Eq. (15) using which a bivariate Gumbel copula given in Eq.(16) can be constructed.

$$
\begin{gathered}
\varphi(t)=(-\ln t)^{\theta} \\
C\left(u_{1}, u_{2}\right)=\exp \left[-\left(\left(-\ln u_{1}\right)^{\theta}+\left(-\ln u_{2}\right)^{\theta}\right)^{1 / \theta}\right\rfloor
\end{gathered}
$$


$\theta$ is the correlation parameter which falls in the range $1 \leq \theta<\infty$, showing perfect independency at $\theta=1$ and perfect dependency as $\theta \rightarrow \infty$.

\subsection{Parameter estimation}

The copula parameter $\theta$ can be estimated with different techniques which include the maximum likelihood (ML) and the Canonical maximum likelihood (CML) methods [13, 18]. For bivariate Archimedean copulas another simplified parameter estimation approach can be used which involves the rank based correlation measures such as Kendall's $\tau$. Kendall's $\tau$ correlation between two random variables $R_{1}$ and $R_{2}$ is computed as the difference between the probability of concordance and probability of discordance of two independent pairs $\left(R_{1}^{l}, R_{2}^{l}\right)$ and $\left(R_{l}^{2}, R_{2}^{2}\right)$.

$$
\tau=\operatorname{Pr}\left\{\left(R_{1}^{1}-R_{1}^{2}\right)\left(R_{2}^{1}-R_{2}^{2}\right)>0\right\}-\operatorname{Pr}\left\{\left(R_{1}^{1}-R_{1}^{2}\right)\left(R_{2}^{1}-R_{2}^{2}\right)<0\right\}
$$

In terms of copula, Kendall's $\tau$ correlation can be written as

$$
\tau=4 \int_{0}^{1} \int_{0}^{l} C\left(u_{1}, u_{2}\right) d C\left(u_{1}, u_{2}\right)-1
$$

For Archimedean copulas the Kendall's $\tau$ correlation coefficient given in Eq. (18) can be written in terms of the generator function and its first derivative as

$$
\tau=1+4 \int_{0}^{l} \frac{\varphi(t)}{\varphi^{\prime}(t)} \delta t
$$

For Gumbel copula Eq. (19) can be simplified in to the following form

$$
\tau=1-\frac{1}{\theta} \quad, 0 \leq \tau<1
$$




\subsection{Goodness-of-fit (GOF)}

Appropriateness of a certain copula for a given data can be checked by Goodness-of-fit (GOF) tests. Several Goodness-of-fit tests have been developed over the years [22-27]. One simple GOF test for bivariate Archimedean copulas can be carried out by comparing the scatter plot between two functions $K_{c}(t)$ and $K_{n c}(t)$ with a straight line having slope $=1$ and $y$-intercept $=$ 0. $K_{c}(t)$ is the parametric function given in Eq. (21) which is the distribution function of a random variable $C(U, V)$ where $(U, V) \in[0,1]^{2}[17]$.

$$
K_{c}(t)=t-\frac{\varphi(t)}{\varphi^{\prime}(t)}
$$

$K_{n c}(t)$ is a non-parametric estimate of the function $K_{c}(t)$ which is obtained as the probability of $t_{i}<t$ for $t_{i}$ defined as follows:

$$
t_{i}=\frac{\text { Number of }\left(u_{i}, v_{i}\right) \text { such that } u_{i}<u_{j} \text { and } v_{i}<v_{j}}{(m-1)} \quad i=1,2, \ldots, m
$$

A copula for which the scatter plot between the two functions better fits to the straight line can be considered to be good for modeling the dependence between the random variables.

\subsection{Multivariate Archimedean copulas}

Deriving mathematical expressions for generator functions and copulas of multivariate distributions having three or more random variables is not obvious as the bivariate case. However, different techniques have been suggested over the years for developing multivariate extensions of Archimedean copulas such as the nested Archimedean construction technique [15, 18, 28]. One method of constructing nested Archimedean copulas is the fully nested approach which is implemented in the present study. This method can be better explained with the help of a schematic illustration for the corresponding mathematical expression gets more complex with 
increasing number of variables. Fig. 1 shows schematic illustration of the fully nested construction of a multivariate Archimedean copula involving four random variables $\left(u_{1}, u_{2}, u_{3}\right.$, u4). As it can be observed, the fully nested construction involves 3 levels of combination. Except the first level where two random variables $u_{1}$ and $u_{2}$ are combined all the remaining levels involve the combination of a copula with a random variable. It is also necessary to make sure that the degree of dependence decreases with increasing level of combination. For instance, parameters of the copulas used at the different levels of the fully nested construction $C_{11}, C_{21}, \ldots$, $C_{31}$ should satisfy the criteria $\theta_{11} \geq \theta_{21} \geq \ldots \geq \theta_{31}$.

\section{Illustrative example}

\subsection{Aerodynamic data}

The building used in the present study is a 50 story steel building which is $182.88 \mathrm{~m}$ (600ft) high with 30.48m (100ft) x 45.72m (150ft) planar dimensions(see Fig. 2(a)). The building has similar dimensions as the Commonwealth Advisory Aeronautical Research Council (CAARC) building [29]. The wind tunnel experiment was conducted at RWDI's boundary layer wind tunnel facility (see Fig. 2(b)). The building model was produced with a 1:400 length scale and tested in open upwind exposure condition whose flow characteristics are shown in Fig. 3. Aerodynamic wind loads on the building are obtained by integrating the simultaneous pressure readings collected with 280 pressure taps installed over the building model surface. Modal analysis of the building model carried out with a finite element software (see Fig. 4) gave the natural frequencies $0.223 \mathrm{~Hz}, 0.367 \mathrm{~Hz}$ and $0.525 \mathrm{~Hz}$ for the first, second and third mode vibrations respectively. The mode shapes corresponding to each mode are also shown in Fig. 5. As it can be seen from the figure the first two modes correspond to sway motions along the $y$ and $x$ axes respectively while the third mode corresponds to a rotational motion about the $z$-axis. A constant damping ratio of $1 \%$ is assumed in all the three vibration modes. The effect of dynamic 
properties of the building is included in the measured aerodynamic data through dynamic analysis carried out in the time domain approach. The peak aerodynamic responses are estimated by a statistical approach which fits normal probability distribution to the time history of windinduced responses [30]. Fig. 6 shows the directional variation of peak base moment coefficients defined as follows:

$$
\begin{gathered}
C M_{s}=\frac{M_{s}}{\frac{1}{2} \rho_{a} \bar{U}_{H}^{2} L H^{2}} \quad s=x, y \\
C M_{\theta}=\frac{M_{\theta}}{\frac{1}{2} \rho_{a} \bar{U}_{H}^{2} L^{2} H}
\end{gathered}
$$

where $H$ is the building height and $L$ is the larger width of the building; $U_{H}$ is the hourly mean wind speed at height $H$; the coefficients $C M_{x}, C M_{y}$ and $C M_{\theta}$ correspond to peak base base moments about the $x, y$ and $z$ axes respectively.

\subsection{Climatological data}

The climatological data used for the present study was taken from the publicly accessible data base of directional wind speeds at the National Institute of Standards and Technology (NIST) website. The data consists of the largest annual 3-sec gust wind speed measured at $10 \mathrm{~m}$ height above the ground in open exposure for a period of 30 years (1950-1979) in Madison, Wisconsin (see Table 1). The 3-sec gust wind speed data was converted into hourly mean wind speed data at the building height so as to make it compatible with the wind tunnel data.

\subsection{Synthesis of aerodynamic and climatological data}

Directional synthesis of the aerodynamic and climatological data was carried out with the sector-by-sector approach. The base moment coefficients obtained from aerodynamic data 
analysis are grouped in eight different sectors and are combined with the corresponding sectorial wind speed vectors. Division and numbering of the different sectors as well as orientation of the building with respect to the wind directions is shown in Fig. 7. Sectorial responses of 700-year MRI are estimated by extreme value analysis using Type I (Gumbel) distribution and the maximum of all sectorial responses are obtained. As discussed previously, the actual MRI corresponding to these maximum moments, however, may or may not be 700 years depending on the type of correlation effect that exists between the sectorial responses. Estimating the actual MRI corresponding to these maximum responses involves combing the sectorial marginal distributions, listed in Table 2, corresponding to these maximum responses. Joint cumulative distribution of the marginal distributions is estimated by Gumbel copula which is selected to be used in the present study using the Goodness-Of-Fit test discussed previously. As it can be seen from Fig. 8, the sample scatter plot of the parametric function $K_{c}(t)$ versus the non-parametric function $K_{n c}(t)$ for the Gumbel copula shows acceptable fit to the $45^{\circ}$ inclined line. Although there are eight different sectors, it may not be necessary to combine all the eight marginals with an eight dimensional Gumbel copula to get the critical joint cumulative distribution. The critical joint cumulative distribution is the minimum probability of non-exceedance which corresponds to the shortest MRI of responses. From practical point of view also it is very unlikely that the extreme effect of a given storm will be manifested in all the eight sectors at the same instant. Hence, it is necessary to decide on the number of sectors required for estimating the critical joint cumulative distribution. Vega [31] stated that considering the contribution of two $45^{\circ}$ sectors or three $22.5^{\circ}$ sectors in directional synthesis of aerodynamic and climatological data is optimal for capturing the required extreme wind effect. In the present study, the joint cumulative distributions obtained by using bivariate Gumbel copula was compared with the results obtained 
from multivariate Gumbel copulas and found to be similar. Hence, considering the combined effect of two $45^{\circ}$ sectors with a bivariate copula was found to be sufficient to get the critical joint cumulative distribution. Bivariate copula analysis was carried out for all possible permutations between two marginals to determine the critical joint cumulative distribution. Table 3 shows the bivariate Gumbel copula parameters obtained for all possible combinations of the sectorial marginal distributions. As it was discussed, the Gumbel copula parameter has a minimum value of 1 which shows perfect independency and as the value approaches $\infty$ perfect dependency will be manifested. Looking at Table 3, the largest correlation effect is observed between sectors 3 and 6 where the largest value of correlation parameter is observed. Using the correlation parameters, the joint cumulative distributions of all possible combinations of the sectorial marginal distributions are obtained (see Table 4). As it can be seen, the critical joint cumulative distribution for $M_{x}$ is observed in sector pair $(6,7)$ and has a value 0.998118 which corresponds to 531-year MRI. Similarly, the critical joint cumulative distribution for $M_{y}$ is observed in sector pair $(6,7)$ and has a value 0.998166 which corresponds to 545 -year MRI. The critical joint cumulative distribution for $M_{\theta}$ is 0.998571 which corresponds to 700-year MRI and it's obtained from all sector pairs where sector 7 is one element in the pair. Occurrence of the critical joint cumulative distribution in multiple sector pairs for $M_{\theta}$ can be explained by looking at the corresponding marginal distributions in Table 3. As it can be seen, all the marginals have a value of 1 except sector 7 which has a value of 0.998571 and this will result in a joint cumulative distribution of 0.998571 based on the first condition of the Sklar's theorem discussed before. It can also be noticed that the critical cumulative distribution may not necessarily occur in the sector pair where the largest correlation effect is observed since the magnitudes of the marginals 
also count besides the correlation effect. For instance, the largest correlation effect occurs in sector pair $(3,6)$ while the critical joint cumulative distribution is observed in sector pair $(6,7)$.

Gumbel copula synthesis is also compared with the traditional simplifying assumptions of perfectly dependent and independent sectorial responses. The MRIs of maximum sectorial responses estimated by combining sectorial marginal distributions with the dependent, independent and Gumbel copulas are listed in Table 5. As it can be observed, the largest MRI values of the $M_{x}$ and $M_{y}$ responses are estimated by the dependent copula while the least MRI values are estimated by the independent copula. The MRI values estimated by Gumbel copula fall in between the values estimated by the dependent and independent copulas. The MRIs of $M_{\theta}$ estimated by all the three copulas are similar as expected.

So far the joint cumulative distribution and MRI values are computed for given values of wind-induced responses. However, what is mostly carried out in a design process is estimating wind-induced responses corresponding to certain MRI values. This can be done by different curve fitting techniques as it is illustrated by the following example. Referring to Table 5, let's consider $M_{x}=1.53 \times 10^{9} \mathrm{Nm}$ which has a 21.1 years MRI estimated by Gumbel copula. Given 21.1 years MRI the corresponding moment response can be estimated by curve fitting through the response values corresponding to the neighboring MRI values 6.9, 14.0, 28.3 and 35.6. Fig. 9 shows the scatter plot of the moment responses versus the MRI values. As it can be seen, the natural logarithm values are plotted rather than the actual values to narrow the gap between successive values and improve accuracy of the curve fit. Accuracy of the fit can also be increased by using more data points close to the point of interest. A third degree polynomial is fitted through the data points using which the value of $M_{x}$ corresponding to 21.1 years MRI is estimated to be $1.53 \times 10^{9} \mathrm{Nm}$. It is advisable to check accuracy of the estimated response by 
computing the MRI value back from the copula using sectorial marginals corresponding to the estimated response value. For this particular example, this may not be necessary since the expected response value is known beforehand (see Table 5).

\section{Conclusion}

Estimation of design wind effects on tall buildings using wind tunnel tests involves integrating the aerodynamic data obtained from wind tunnel tests with the actual climatological data. Sector-by-sector approach is one of the synthesis approaches used for integrating aerodynamic and climatological data. Simplicity of this method compared to most existing techniques makes it attractive for practical application. However, the inability of properly considering the correlation effect between sectorial responses has always been a challenge for comfortably applying the method. Recognizing this challenge, the present study introduces a new approach for modeling this correlation effect between sectorial responses. The study showed that a copula based approach can be used for accurate modeling of the correlation effect between sectorial responses. Application of the proposed approach was illustrated with a numerical example on a 50 story building. In addition, the proposed approach was used to assess the uncertainties in the traditionally used simplifying assumptions of perfectly dependent or independent sectorial responses while implementing the sector-by-sector approach. Results of the study showed that assuming sectorial responses to be perfectly dependent or independent could result in underestimation or overestimation of design wind effects respectively. It was also

noted that it is possible to come across a case where assuming perfect dependency and independency could both give fairly close results. Thus, while using the sector-by-sector approach, it is a good place to start by comparing results obtained from assuming perfect dependency with the results obtained from perfect independency. If the results obtained from the two simplifying assumptions are significantly different, the copula based approach can be used to 
accurately model of the correlation effect between sectorial responses. When the results obtained from the two simplifying assumptions are close enough, however, it may not be necessary to implement the copula based approach as it won't produce significantly different results.

\section{Acknowledgment}

This research is based upon work supported by the National Science Foundation under Grant No. 0846811, and Canada Research Chair and Ontario Early Career Awards to the second author. Any opinions, findings, and conclusions or recommendations expressed in this material are those of the authors and do not reflect the views of the granting agencies.

\section{References}

[1] Cook NJ. Towards estimation of extreme winds. J Wind Eng Ind Aerodyn. 1982;9:295-323.

[2] Moriarty WW, Templeton JI. On the estimation of extreme wind gusts by direction sector. J Wind Eng Ind Aerodyn. 1983;13:127-38.

[3] Heckert NA, Simiu E, Whalen T. Estimates of hurricane wind speeds by "peak over threshold" method. J Struct Eng. 1998;124.

[4] Rigato A, Chang PC, Simiu E. Database-assisted design and wind directionality effects. Bluff body aerodynamics and applications, $4^{\text {th }}$ International colloquium. Ruhr-University Bochum 2000. p. 213-6.

[5] Spence SMJ. Time domain non-Gaussian optimization of wind excited tall buildings under vulnerability constraints [phd dissertation]: University of Braunschweig and University of Florence; 2009.

[6] Rigato A, Chang P, Simiu E. Database-Assisted Design, standardization, and wind direction effects. J Struct Eng. 2001;127:855-60.

[7] Simiu E, Gabbai RD, Fritz WP. Wind-induced tall building response:a time-domain approach. Wind and Structures. 2008;11:427-40.

[8] Davenport AG. The prediction of risk under wind loading. $2^{\text {nd }}$ International conference on structural safety and reliability. Munich, Germany 1977. p. 511-38.

[9] Lepage MF, Irwin PA. A technique for combining historical wind data with wind tunnel tests to predict extreme wind loads. Proceedings of the $5^{\text {th }}$ US national conference on wind engineering Texas Tech University, Lubbock, Texas 1985.

[10] Irwin P, Garber J, Ho E. Integration of wind tunnel data with full scale wind climate. 10th Americas conference on wind engineering. Baton Rouge, LA 2005.

[11] Simiu E, Miyata T. Design of Buildings and Bridges for Wind. Hoboken, New Jersey: John Wiley Sons; 2006.

[12] Gamble SL, Miltenburg RJ, Cicci MD, Accardo M. Prediction of local exterior wind pressures from wind tunnel studies using a time history analysis approach. Proceedings of the Americas conference on wind engineering. Baltimore 2001.

[13] Trivedi PK, Zimmer DM. Copula Modeling: An Introduction for Practitioners. Foundations and Trends in Econometrics: Now Publishers; 2007. p. 111.

[14] Schmidt T. Coping with Copulas. Copulas - From Theory to Applications in Finance. UK: Risk Books; 2007. p. 3-34.

[15] McNeil AJ, Frey R, Embrechts P. Quantitative Risk Management: Concepts, Techniques and Tools. Princeton, New Jersey: Princeton University Press; 2005. 
[16] Embrechts P, McNeil AJ, Straumann D. Correlation and dependence in risk management: properties and pitfalls. Cambridge: Cambridge University Press; 2002.

[17] Nelsen RB. An Introduction to Copulas. second ed. New York: Springer-Verlag; 2006.

[18] Joe H. Multivariate Models and Dependence Concepts. London: Chapman \& Hall; 1997.

[19] Sklar A. Fonctions de répartition à $\mathrm{n}$ dimensions et leurs marges. Publ Inst Statist Univ Paris 1959;8:229-31.

[20] Fréchet M. Les tableaux de corrélation dont les marges et des bornes sont données. Annales de l'Université de Lyon, Sciences Mathématiques et Astronomie. 1957;20:13-31.

[21] Embrechts P, Lindskog F, McNeil A. Modelling Dependence with Copulas and Applications to Risk Management. Zürich, Switzerland: Department of Mathematics ETHZ CH-8092; 2001.

[22] Berkowitz J. Testing density forcasts, with applications to risk management. Journal of Business and Economic Statistics. 2001;19:465-74.

[23] Andersen PK, Ekstrøm CT, Klein JP, Shu Y, Zhang M-J. A class of goodness of fitt tests for a copula based on bivariate right-censored data. Biometrical Journal. 2005;47:815-24.

[24] Genest C, Rivest L-P. Statistical inference procedures for bivariate archimedean copulas. Journal of the American Statistical Association. 1993;88:1034-43.

[25] Genest C, Quessy J-F, Rémillard B. Goodness-of-fit procedures for copula models based on the probability integral transform. Scandinavian Journal of Statistics. 2006;33:337-66.

[26] Wang W, Wells MT. Model selection and semiparametric inference for bivariate failure-time data. Journal of the American Statistical Association. 2000;95:62-72.

[27] Diebold FX, Gunther TA, Tay AS. Evaluating density forcasts, with applications to financial risk management. International Economic Review. 1998;39:863-83.

[28] Whelan N. Sampling from Archimedean copulas. Quantitative Finance. 2004;4:339-52.

[29] Melbourne WH. Comparison of measurements on the CAARC standard tall building model in simulated model wind flows. J Wind Eng Ind Aerodyn. 1980;6:73-88.

[30] Warsido W, Bitsuamlak G. Estimating peak wind-induced responses of tall buildings. 2012 joint Conference of the Engineering Mechanics Institute and 11th ASCE Joint Speciality Conference on Probabilistic Mechancis and Structural Reliability. Notre Dame, IN 2012.

[31] Vega RE. Wind directionality: A reliability-based approach [phd dissertation]: Texas Tech University; 2008. 
Table $1.3 \mathrm{sec}$ gusts in $\mathrm{m} / \mathrm{s}$ based on original records at $10 \mathrm{~m}$ height for Madison, WI

\begin{tabular}{lllllllll}
\hline Year & N & NE & E & SE & S & SW & W & NW \\
\hline 1950 & 23 & 27 & 23 & 25 & 15 & 40 & 38 & 25 \\
1951 & 21 & 19 & 24 & 17 & 20 & 38 & 24 & 38 \\
1952 & 25 & 20 & 16 & 18 & 21 & 25 & 23 & 20 \\
1953 & 26 & 20 & 24 & 23 & 33 & 25 & 29 & 24 \\
1954 & 19 & 17 & 22 & 15 & 17 & 36 & 24 & 23 \\
1955 & 23 & 19 & 22 & 20 & 18 & 26 & 25 & 24 \\
1956 & 19 & 24 & 20 & 17 & 20 & 23 & 25 & 23 \\
1957 & 18 & 19 & 17 & 23 & 20 & 21 & 30 & 21 \\
1958 & 20 & 21 & 20 & 15 & 20 & 24 & 23 & 19 \\
1959 & 20 & 23 & 15 & 19 & 20 & 21 & 25 & 23 \\
1960 & 18 & 23 & 17 & 23 & 20 & 30 & 20 & 23 \\
1961 & 19 & 23 & 20 & 20 & 25 & 24 & 22 & 19 \\
1962 & 22 & 20 & 18 & 21 & 20 & 24 & 30 & 19 \\
1963 & 31 & 20 & 15 & 20 & 20 & 28 & 20 & 24 \\
1964 & 22 & 18 & 16 & 17 & 24 & 28 & 27 & 33 \\
1965 & 20 & 19 & 21 & 19 & 19 & 30 & 21 & 21 \\
1966 & 17 & 17 & 19 & 16 & 27 & 19 & 21 & 23 \\
1967 & 18 & 17 & 22 & 17 & 20 & 25 & 21 & 22 \\
1968 & 19 & 19 & 17 & 20 & 21 & 22 & 31 & 21 \\
1969 & 20 & 21 & 13 & 20 & 18 & 22 & 17 & 20 \\
1970 & 17 & 16 & 23 & 23 & 19 & 22 & 22 & 32 \\
1971 & 20 & 21 & 19 & 17 & 19 & 24 & 20 & 23 \\
1972 & 18 & 22 & 18 & 21 & 19 & 21 & 20 & 24 \\
1973 & 18 & 21 & 16 & 17 & 20 & 19 & 19 & 27 \\
1974 & 21 & 17 & 17 & 17 & 21 & 22 & 17 & 21 \\
1975 & 17 & 21 & 22 & 19 & 18 & 26 & 25 & 20 \\
1976 & 26 & 20 & 12 & 16 & 17 & 19 & 17 & 21 \\
1977 & 22 & 17 & 17 & 17 & 17 & 22 & 26 & 20 \\
1978 & 19 & 17 & 15 & 19 & 17 & 21 & 17 & 20 \\
1975 & 18 & 17 & 23 & 17 & 18 & 26 & 17 & 26 \\
\hline & & & & & & & & \\
\hline
\end{tabular}


Table 2 Marginal CDFs in different sectors corresponding to given values of $M_{x}, M_{y}$ and $M_{\theta}$

\begin{tabular}{c|cccccccc}
\hline & \multicolumn{8}{c}{ Sector } \\
\cline { 2 - 9 } Moment $\left(\mathrm{x} 10^{9} \mathrm{Nm}\right)$ & 1 & 2 & 3 & 4 & 5 & 6 & 7 & 8 \\
\hline$M_{x}=2.43$ & 0.999995 & 1.000000 & 1.000000 & 1.000000 & 0.999975 & 0.999264 & 0.998571 & 0.999917 \\
$M_{y}=1.30$ & 0.999997 & 1.000000 & 1.000000 & 1.000000 & 0.999968 & 0.998571 & 0.999328 & 0.999929 \\
$M_{\theta}=0.14$ & 1.000000 & 1.000000 & 1.000000 & 1.000000 & 1.000000 & 0.999999 & 0.998571 & 1.000000 \\
\hline
\end{tabular}


Table 3 Bivariate Gumbel copula parameter $\left(\theta_{i j}\right)$ between sectors $i$ and $j$.

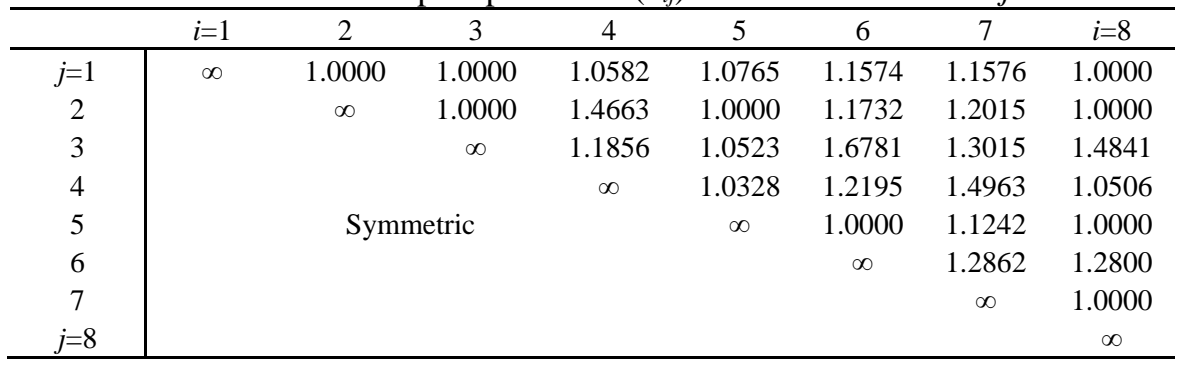


Table 4 Joint CDF between sectors $i$ and $j$

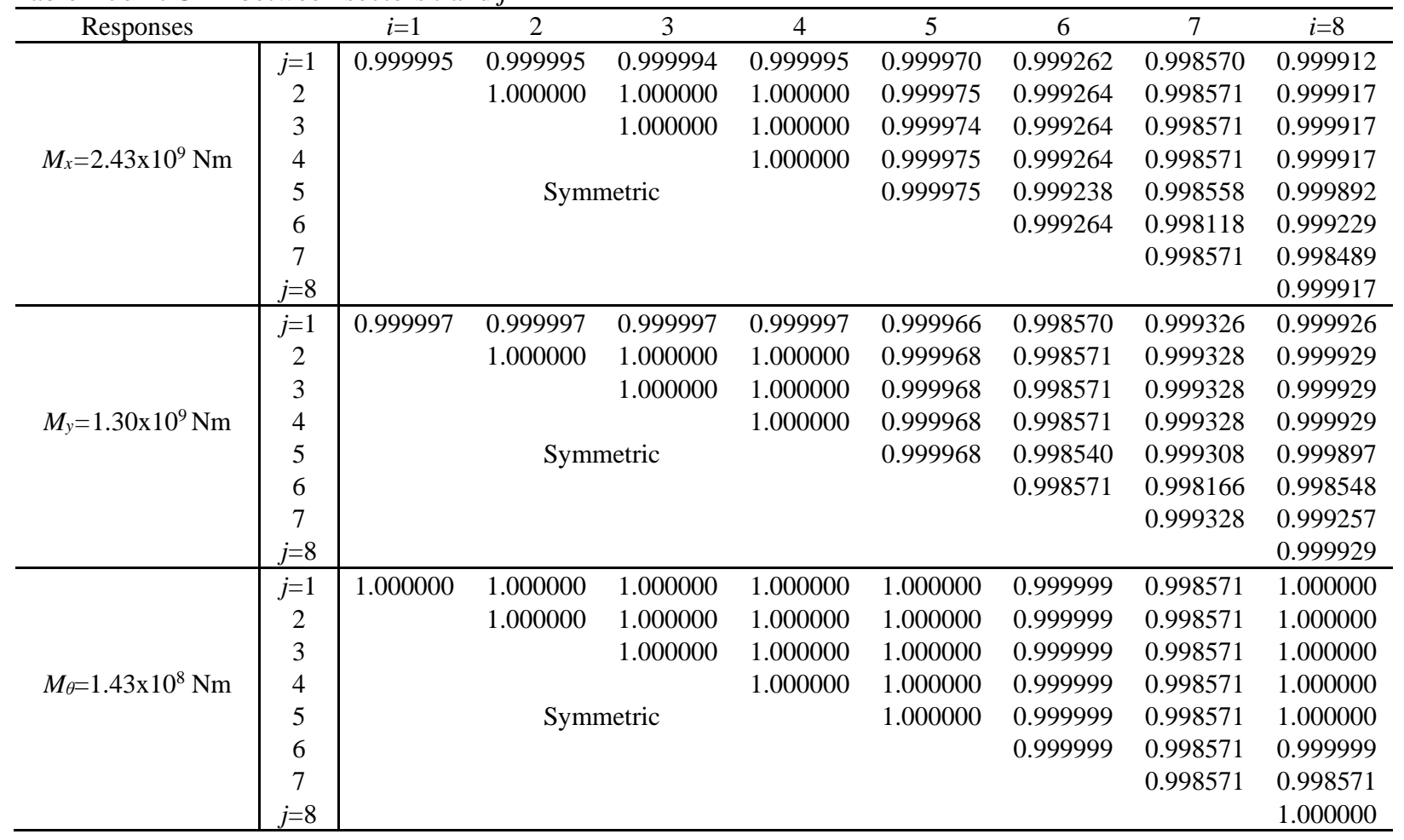


Table 5 MRI of moment base moments in years estimated by different copulas

\begin{tabular}{cc|ccc}
\hline Responses & & Dependent & Independent & Gumbel \\
\hline & 1.21 & 10.0 & 4.6 & 6.9 \\
& 1.42 & 20.0 & 9.8 & 14.0 \\
& 1.53 & 30.0 & 15.3 & 21.1 \\
$M_{x}\left(\times 10^{9} \mathrm{Nm}\right)$ & 1.62 & 40.0 & 20.9 & 28.3 \\
& 1.68 & 50.0 & 26.6 & 35.6 \\
& 1.88 & 100.0 & 56.1 & 72.4 \\
& 2.33 & 500.0 & 308.6 & 376.5 \\
& 2.43 & 700.0 & 439.0 & 531.3 \\
& 2.53 & 1000.0 & 637.3 & 765.1 \\
\hline & 0.65 & 10.0 & 4.7 & 7.1 \\
& 0.76 & 20.0 & 10.1 & 14.3 \\
& 0.82 & 30.0 & 15.7 & 21.6 \\
& 0.86 & 40.0 & 21.5 & 29.0 \\
$M_{y}\left(\times 10^{9} \mathrm{Nm}\right)$ & 0.90 & 50.0 & 27.4 & 36.4 \\
& 1.00 & 100.0 & 57.8 & 74.2 \\
& 1.25 & 500.0 & 318.5 & 386.3 \\
& 1.30 & 700.0 & 453.4 & 545.3 \\
& 1.35 & 1000.0 & 658.3 & 785.5 \\
\hline & 0.72 & 10.0 & 9.6 & 10.0 \\
& 0.84 & 20.0 & 19.5 & 20.0 \\
$M_{\theta}\left(\times 10^{8} \mathrm{Nm}\right)$ & 0.90 & 30.0 & 29.5 & 30.0 \\
& 0.95 & 40.0 & 39.5 & 40.0 \\
& 0.10 & 50.0 & 49.5 & 50.0 \\
& 1.11 & 100.0 & 99.5 & 100.0 \\
& 1.38 & 500.0 & 499.8 & 500.0 \\
& 1.43 & 700.0 & 699.5 & 1000.0 \\
\hline 1.49 & 1000.0 & 999.5 &
\end{tabular}




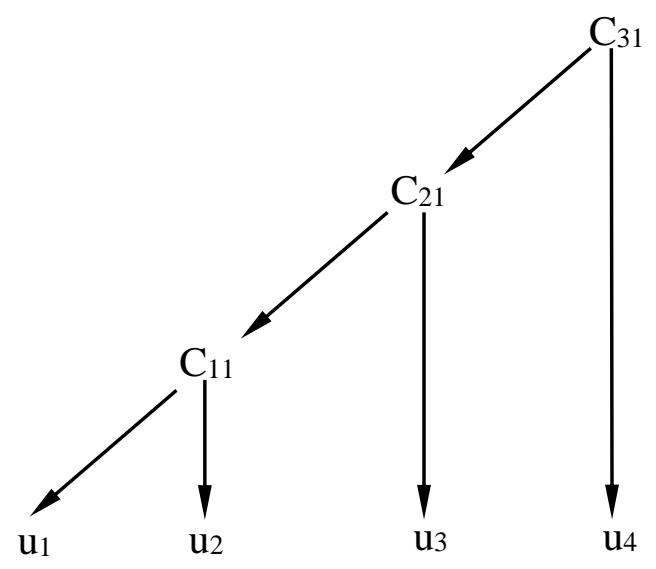

Fig. 1 Schematic illustration of the fully nested construction of a multivariate Archimedean copula with four random variables 

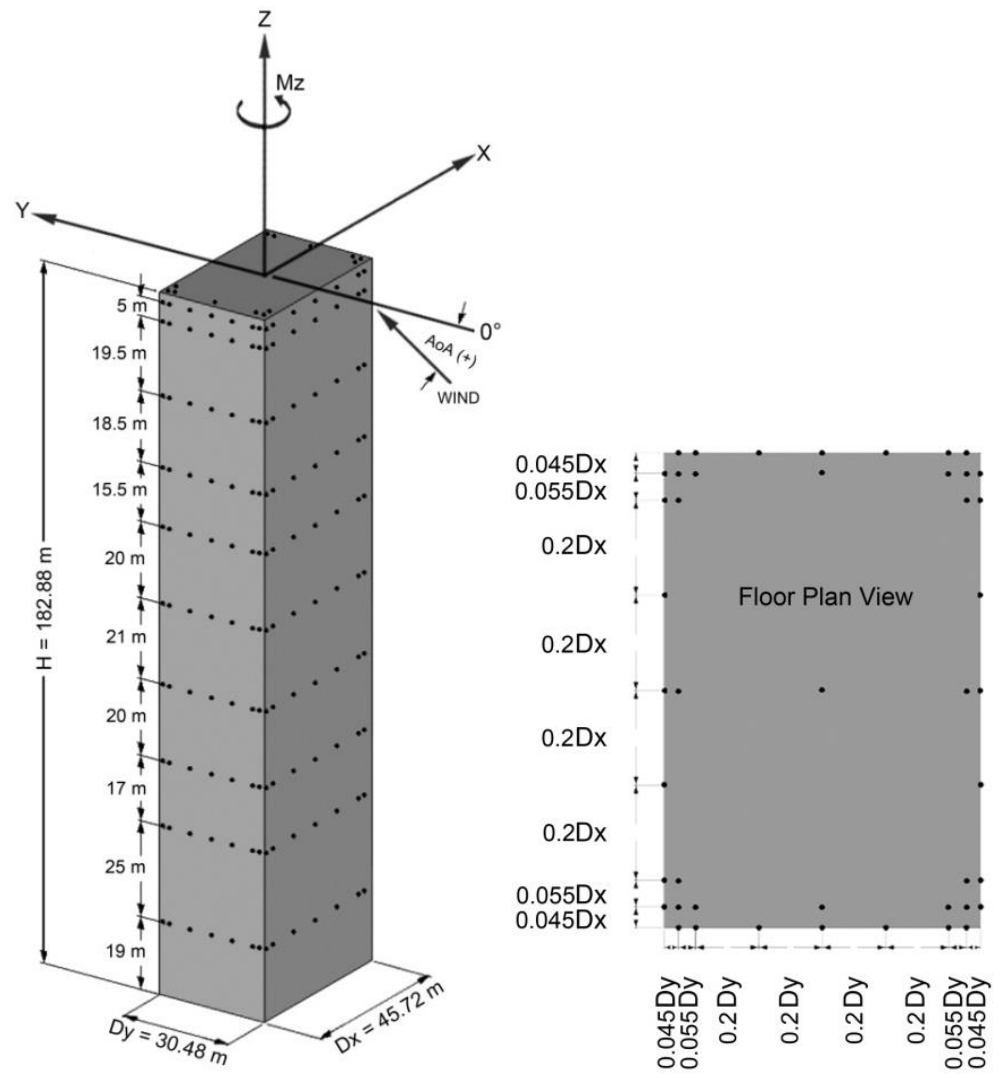

(a)

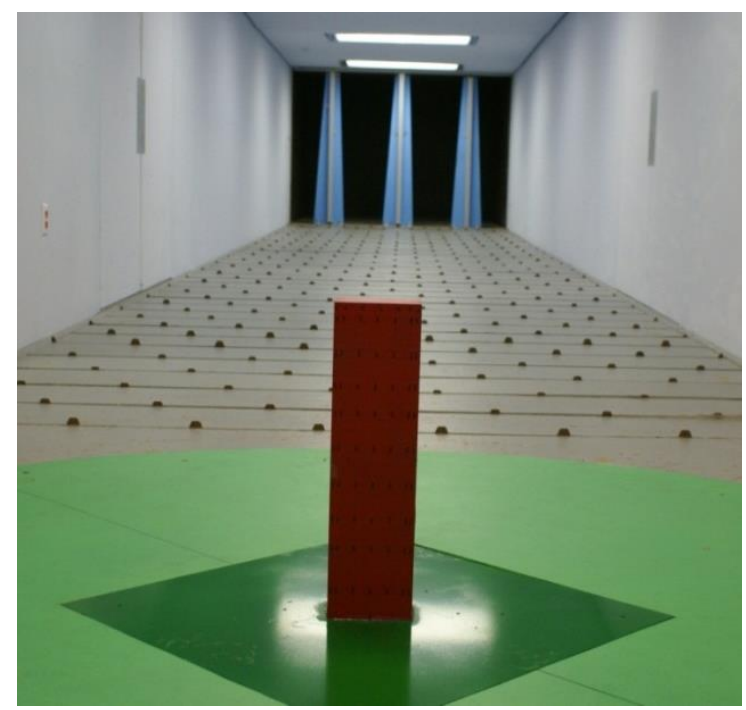

(b)

Fig. 2 (a) Dimensions of the study building in $m$ and pressure tap locations; (b) HFPI test setup at RWDI's boundary layer wind tunnel laboratory 

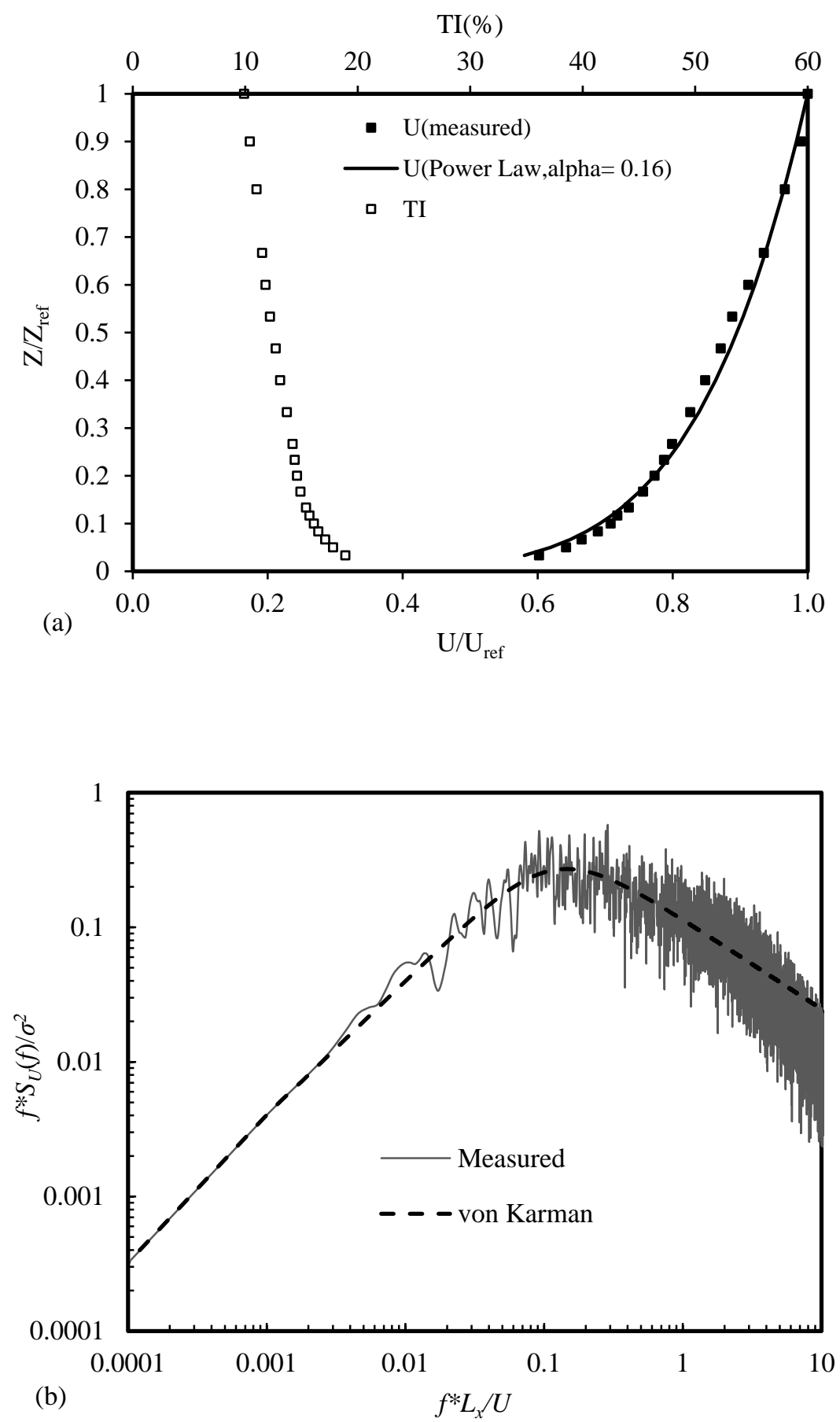

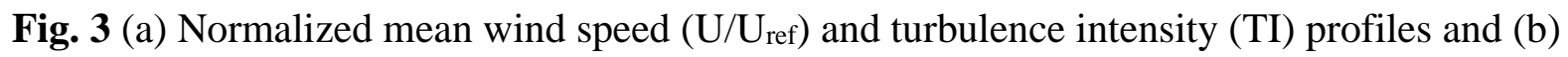
longitudinal turbulence spectrum at the building height 


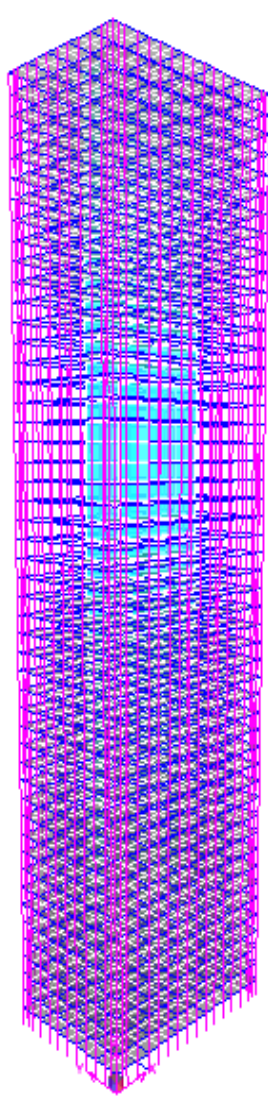

(a)

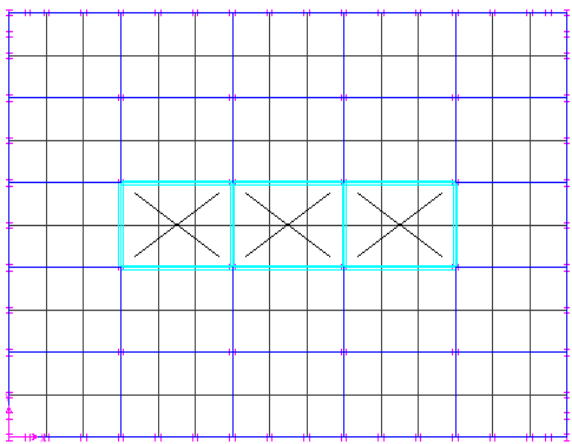

(b)

Fig. 4 FEM model of the study building: (a) 3D view and (b) typical plan view 

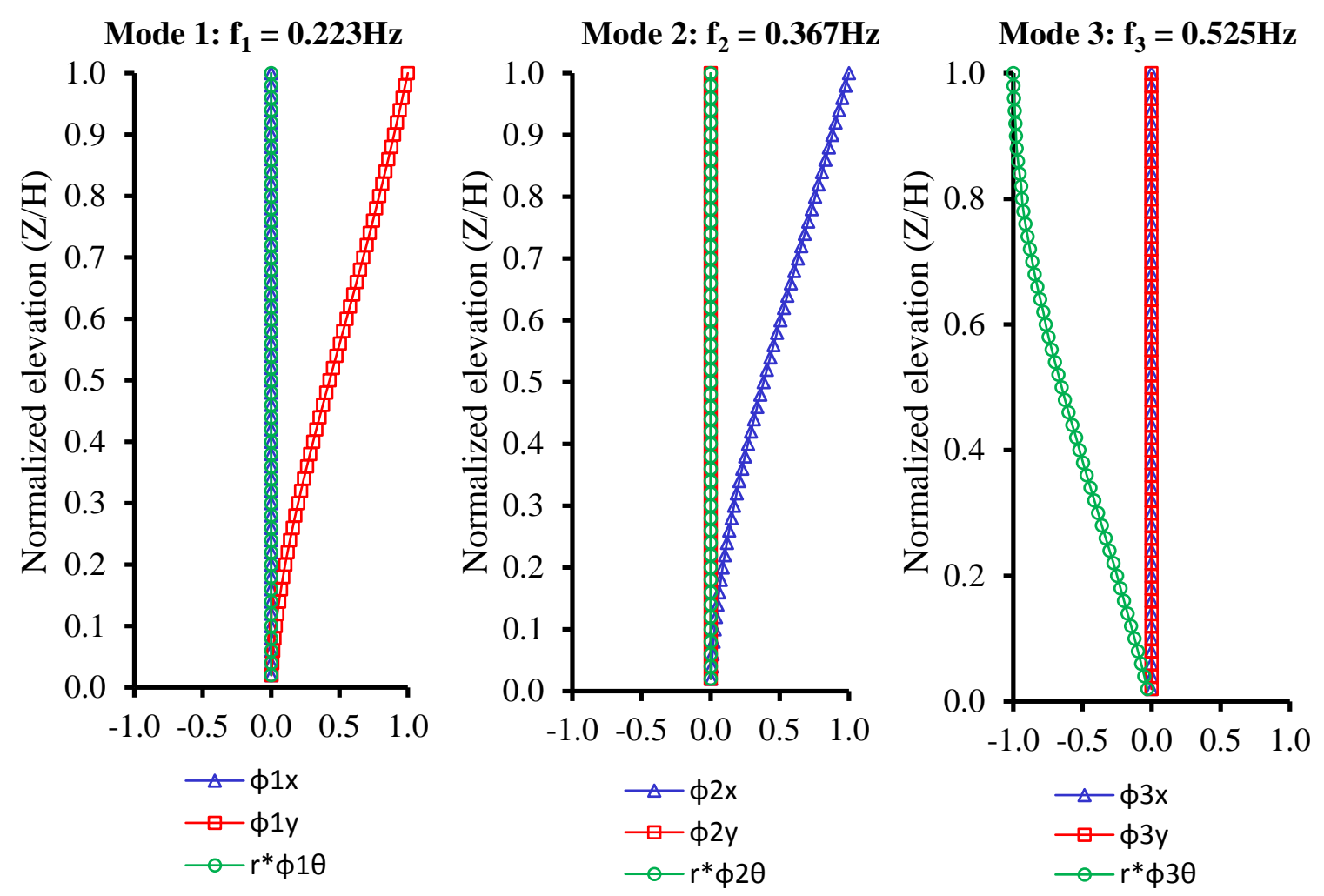

Fig. 5 Normalized (a) first, (b) second and (c) third vibration modes 


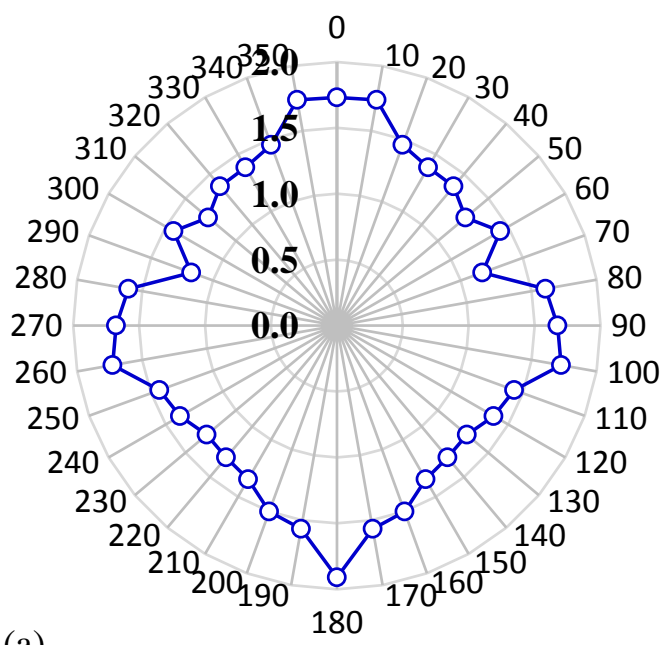

(a)
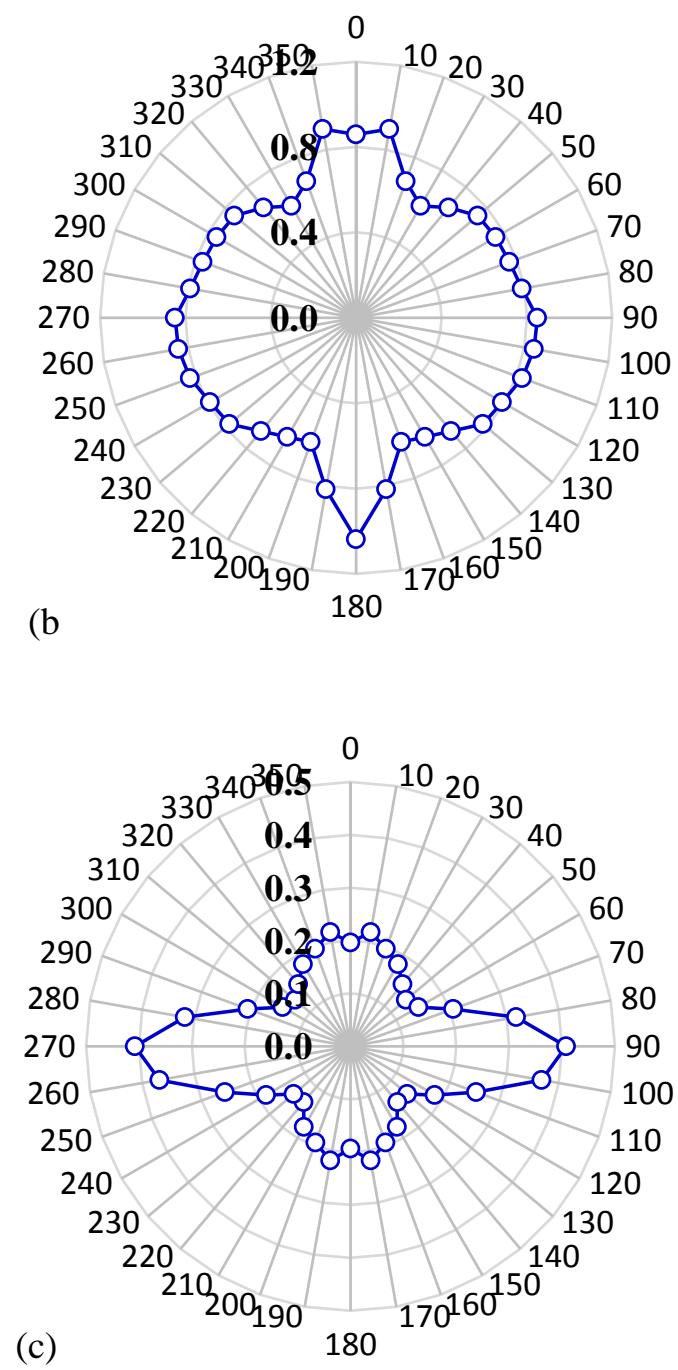

Fig. 6 Peak base moment coefficients (a) $C M x$, (b) $C M_{y}$ and (c) $C M_{\theta}$ from wind tunnel testing 


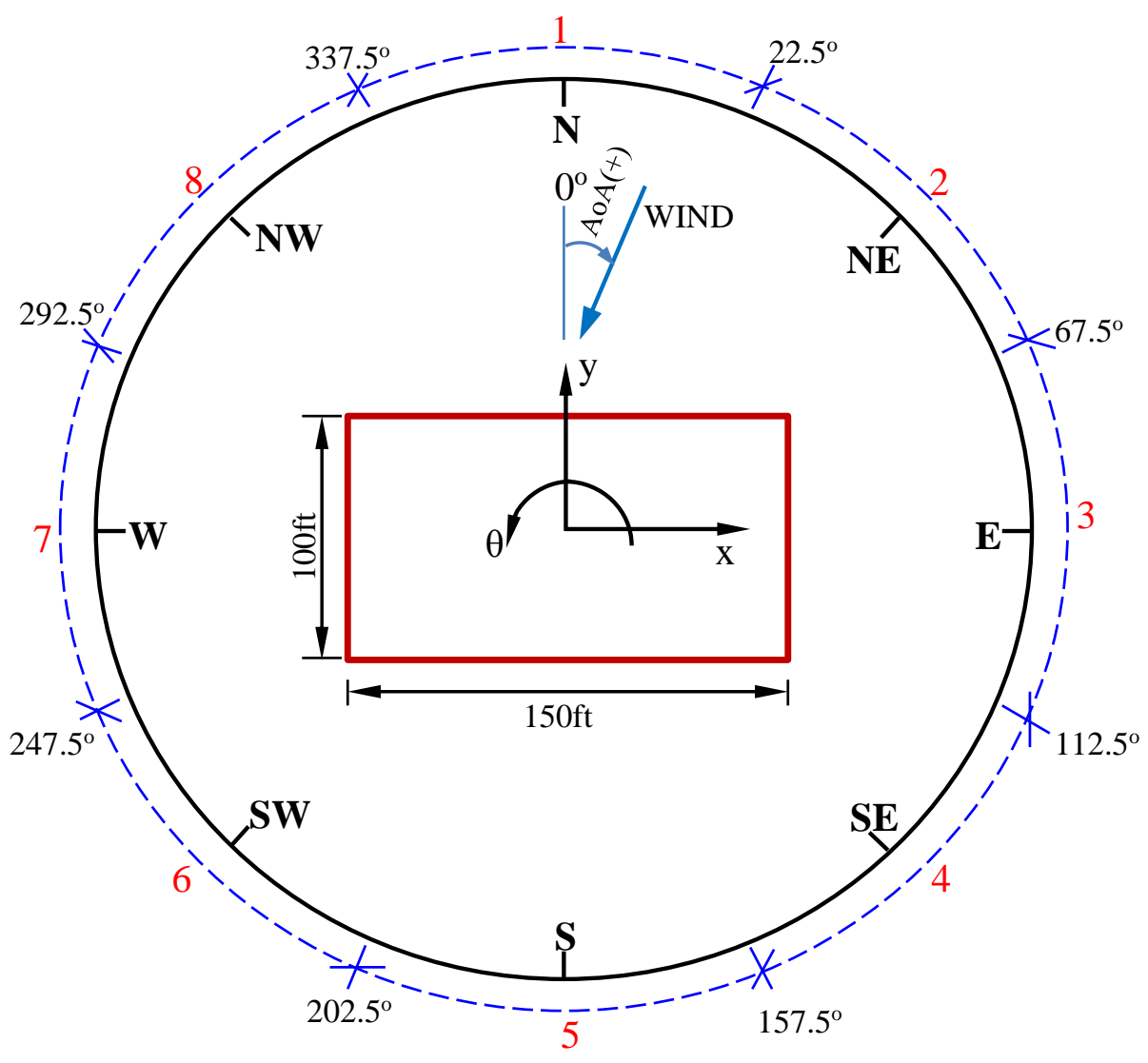

Fig. 7 Orientation of the study building with respect to wind direction and sectorial division for directional synthesis 


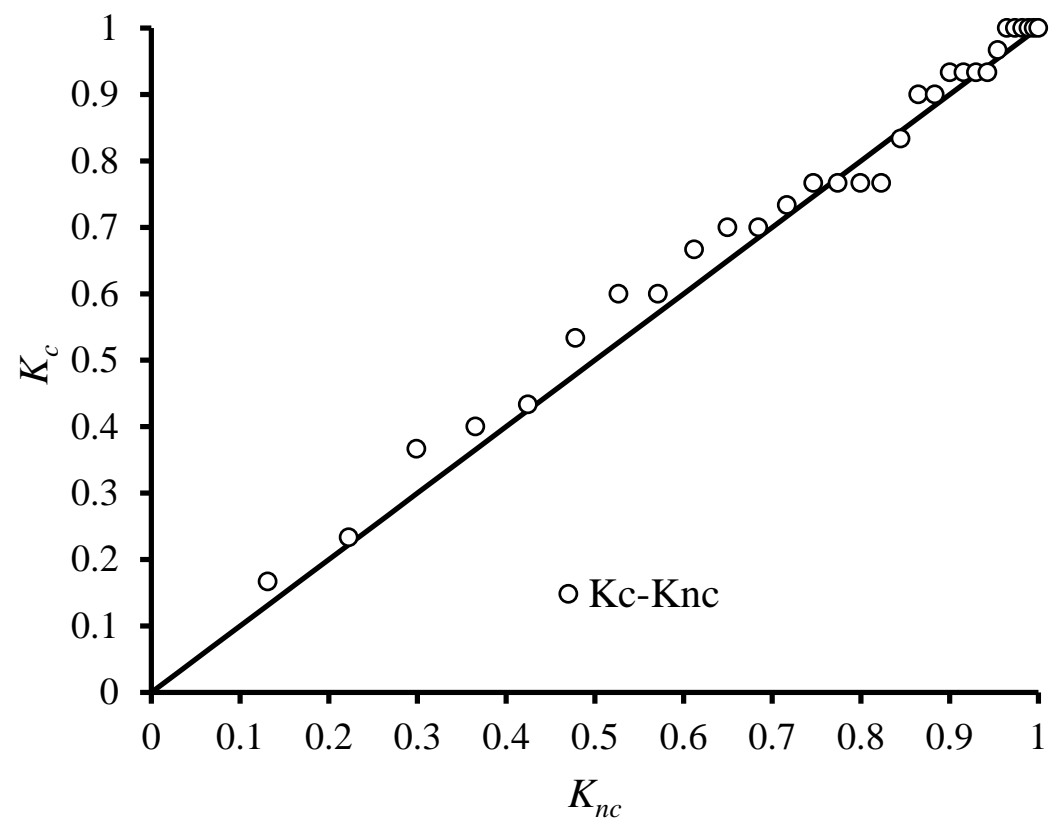

Fig. 8 Plot of parametric function $K_{c}$ versus non-parametric function $K_{n c}$ 


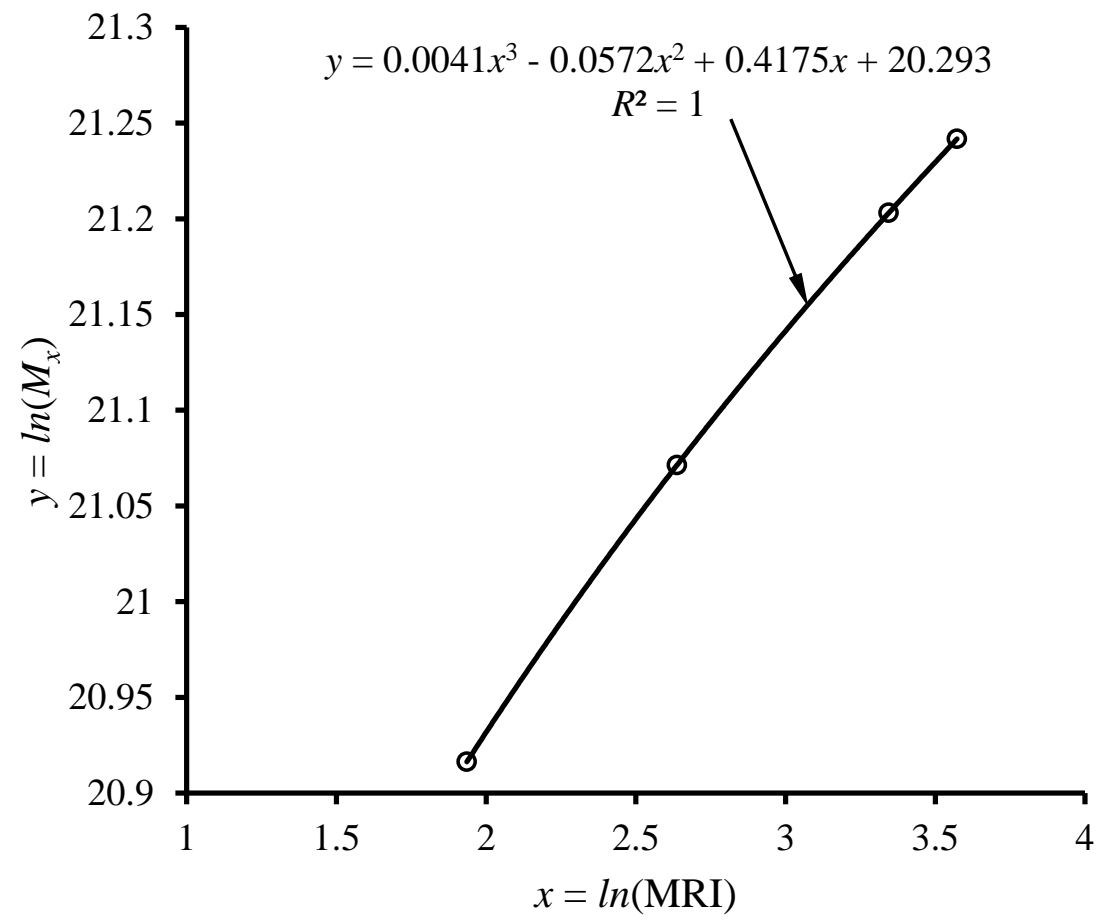

Fig. 9 Variation of base moment $M_{x}$ with mean recurrence interval (MRI) 